\title{
Role of Xuesaitong in Amelioration of Neural Function and Alteration of Bax Expression in Rats with Brain Trauma
}

\author{
Wei Huang1*, Qi Yan²*, Jia Liư3 , Jintao Li"\#, Tinghua Wang4"\# \\ ${ }^{1}$ Neurology Department of the 3rd People's Hospital of Yunnan Province, Kunming, China \\ ${ }^{2}$ No. 2 Neurosurgery Department of the 1st Hospital of Kunming Medical University, Kunming, China \\ ${ }^{3}$ Experimental Animal Center of Kunming Medical University, Kunming, China \\ ${ }^{4}$ Neuroscience Institute of Kunming Medical University, Kunming, China \\ Email: 1972489116@qq.com, kmyanqi@126.com, liujiaaixuexi@126.com, "kmlijintao@163.com, \\ "tinghua neuron@263.net
}

Received 6 July 2015; accepted 20 September 2015; published 23 September 2015

Copyright (C) 2015 by authors and Scientific Research Publishing Inc.

This work is licensed under the Creative Commons Attribution International License (CC BY).

http://creativecommons.org/licenses/by/4.0/

(c) (i) Open Access

\begin{abstract}
Traumatic brain injury (TBI) is one of the most common diseases in clinical neurosurgery characterized with high incidence rate, mortality and many complications. Objectives: The purpose of this study is to explore the roles of Xuesaitong in the therapeutic effect of brain trauma and alteration of expression in Bax, a kind of promoting apoptosis factor. Methods: The rat traumatic brain injury models were established by using modified free falling body impact method. Thereafter, Xuesaitong was employed to be administered to TBI rats, and NSS Score Rating Scale was used to evaluate the effect of Xuesaitong. Moreover, real-time PCR was used to detect the Bax expression changes before and after the Xuesaitong administration. Results: Xuesaitong could accelerate the neurofunctional recovery of TBI rats, accompanied by NSS Scores significant decrease. Simultaneously, it also could inhibit the expression of Bax factor. Conclusions: Xusaitong could markedly ameliorate TBI restoration, in which it promotes the neurofunctional recovery, and at the same time it inhibits the expression of Bax.
\end{abstract}

\section{Keywords}

Traumatic Brain Injury, Neurofunction, Xuesaitong Injections, Bax

\footnotetext{
"Wei Huang and Qi Yan contributed equally to this work.

${ }^{\#}$ Corresponding authors. They contributed equally to this work.
}

How to cite this paper: Huang, W., Yan, Q., Liu, J., Li, J.T. and Wang, T.H. (2015) Role of Xuesaitong in Amelioration of Neural Function and Alteration of Bax Expression in Rats with Brain Trauma. International Journal of Clinical Medicine, 6, 682-689. http://dx.doi.org/10.4236/ijcm.2015.69091 


\section{Introduction}

Traumatic Brain Injury (TBI) is one of the most common diseases in neurosurgery with high incidence rate, mortality and muti-complications. It could result in movement defect, cognition disorder, impairment of language function. The lesion of TBI also includes direct injury occurring at the injury site and secondary injury induced by the pathological process including ischemia, anoxia, calcium channel dysfunction and lipid peroxidation [1]. Among all the complications, cognition disorder is the most sustained and serious symptom, which expressed as juries or loss of attention and memory [1] [2]. While local brain ischemia is one of the main causes of secondary craniocerebral injury, $90 \%$ among which is the direct cause to death [3]. Currently, to our knowledge, there are several following methods dedicated to clinical therapy for TBI: cryotherapy, hormonal therapy, neurotrophic factors and growth factors therapies, cellular transplanting therapies (such as Bone Marrow Stem cells (BMSC) and Neural Stem Cells (NSCs)) [2].

The main component of Xuesaitong freeze-dry powder for injection is Panax Notoginsenosidum (PNS) extracted from Panax Notoginseng (Sanqi), which is a kind of famous traditional Chinese Medicine. It has various effects of activating blood circulation to dissipate blood stasis, promoting blood circulation and activating collaterals, so it can stretch blood vessels, inhibit the platelet aggregation, reduce the content of fibrinogen in the blood flow and blood viscosity, thereby ameliorate the microcirculation and exert pharmacologic actions of antiinflammation and anti-oxidant [4]. All these accelerate the absorption of hematoma, promote the fadeaway of tissue edema surrounding the injury sits and ultimately improve the recovery of neural dysfunction [5].

Xuesaitong freeze-dry powder for injection used in this study is off-white to stramineous color that is powder in amorphous forms or loosened solid powder with bitter in taste and subtle sweet. It can relieve brain edema and inflammatory reaction so as to promote the absorption of intracranial hematoma [6]. Xuesaitong is a new type of thrombolysis anticoagulant, which has been widely used in clinic practice due to its beneficial effects on raising the cerebral blood flow, ameliorate the blood circulation of infarction zone and effectively prohibit the formation of thrombus. It exerts effects on brain protection under injured or anoxic condition through modifying blood circulation, taking against platelet aggregation and prolonging Clotting time [7].

Bax is an apoptosis promoted factor that commonly present in the cytoplasm as transducer in cellular damage and stimulation. However, when cellular apoptosis occurs, Bax that associates with mitochondrial may present in a manner of monomer without the activity. Or it may present in a manner of activated complex with big molecular weight that combined with mitochondria membrane [8] [9]. Responded to injury and stimulation, Bax will re-localize at surface of mitochondrial and further destroy the function of Bcl-2 protein that has anti-apoptotic effect in normal condition.

During the process of apoptosis, Bcl-2 gene family plays a critical role [10]. Bcl-2 gene family is divided into two kinds of protein-anti-apoptotic protein and promoting apoptotic protein. The product of Bax, an important factor attached to promoting apoptotic protein [11], has an effect on antagonize the bioactivity of bcl-2, which is homologous to Bax's product, an associated protein of bcl-2. Bax could form a bore spanning the mitochondrial outer membrane, resulting in reduction of the membrane potentials and outflow of Cytochrome $\mathrm{C}$ (Cyt C) and apoptosis inducing factor (AIF). Whereas Cyt C, combining with Apaf-1, ATP and pro-Caspase-9, form apoptosome compound, which activates Caspase-9. When binding to Cyt $\mathrm{C}$, the combing capacity between Apaf-1 and pro-Caspase-9 was strengthened. Anti-apoptotic molecule Bcl-2 and Bcl-XL play a role in prohibiting the bore formation on the mitochondrial. When Bax, Bcl-2 and Bcl-XL form heterodimer, it blocks up the anti-apoptotic effects exerted by these molecules [7]. These anti-apoptotic effects may attributed to the underlying mechanism by destroying the mitochondrial outer membrane, promoting the assembly of death-inducing signing complex (DISC) and activity of Caspase-3, Caspase-8, ultimately playing roles in promoting apoptosis [11]. It has been demonstrated that expression of Bax closely associated with incidence and development of various malignant tumors [12] [13]. Moreover, it has been revealed that Bax could mediate the apoptotic sensibilization produced by fibroin of mammary gland. This provides new foundation for the treatment of breast cancer and prostatic cancer [14], suggesting Bax may play a crucial role in clinical tumor therapy. However, to our knowledge, the relationship between Bax and TBI is few reported and largely unknown.

Taken together, traumatic brain injury (TBI) model was employed in this study. NSS Score System was used to evaluate the therapeutic effect of Xuesaitong. Furthermore, qPCR was used for Bax expression detection. And the underlying action mechanism of Xuesaitong was investigated. Additionally, the relationship of Bax expression level and Xuesaitong's effects on TBI rats was elucidated. 


\section{Methods}

\subsection{Animal and Grouping}

A total of 30 Sprague Dawley (SD) rats, weighing 200 - 240 g, provided by Experimental Animal Center of Kunming Medical University, were used in this study. The animal grouping was shown in Table 1. TBI group was TBI rats without Xuesaitong injection. Sham group was rats subjected to craniotomy and cerebral dura mater exposure only. Rats in TBI-Xuesaitong injection group underwent TBI + Xuesaitong injection. In TBI + Xuesaitong group, rats were fixed and Xusaitong solution (200 mg/bottle, Pharmaceutical Group co.ltd of Kunming, China) was injected at 8 am every morning via caudal vein daily from 1 to $14 \mathrm{~d}$ after operation (200 mg/d).

\subsection{Animal Model Preparation}

The rat traumatic brain injury model was prepared by using weight free drop impact method [15] [16]. Briefly, SD rats were anesthetized intraperitoneally with $3.6 \%$ chloral hydrate $(1 \mathrm{ml} / 100 \mathrm{~g})$. And then, rats were fixed in prone position. An incision was performed along the sagittal plane of the left parietal bone of skull, the left parietal bone was exposed. A $5.0 \mathrm{~mm} \times 5.0 \mathrm{~mm}$ sized bone window was formed at approximately $2.5 \mathrm{~mm}$ aside from sagittal suture and $1.5 \mathrm{~mm}$ aside from coronal suture, the cerebral dura mater was then exposed. Subsequently, an iron midheaven cylinder, weighing $59 \mathrm{~g}$, was allowed to drop from $10 \mathrm{~cm}$ height along with a metal pole. When it impacted on the clout placed on the surface of cerebral cortex, the contusion of left motor cortex formed, which could mimic the state of traumatic brain injury. Following the operation, the foods and water was ad libitum. Penicilline sodium $(0.5 \mathrm{ml} / 20 \mathrm{IU})$ was injected im. to prevent infection.

\subsection{Neurological Severity Score Evaluation [17] [18]}

Neurological Severity Scores (NSS) Rating Scale System was used to evaluate the motor function of rats following TBI. NSS assessment was conducted daily by blindness method from Day 1 to Day 14 after operation. The NSS Scales was shown in Table 2. SPSS software was employed to analyze the data.

The score ranged from 2 - 18. 13 - 18 score represented severe injury. 7 - 12 score represented moderate injury. While 1 - 6 score represented mild injury [19]. NSS Score also was used to evaluate whether or not the TBI rat models was installed successfully.

\subsection{Real-Time RT-PCR}

The brain tissues of rats were harvested from rats in all experimental groups on 1d post of TBI. Total RNA was isolated from brain tissues using Trizol reagent (Invitrogen) to produce cDNA, respectively. Then, quantitative RT-PCR (qRT-PCR) analysis was performed to analyze the level of Bax. QRT-PCR of cDNA was performed, and then the PCR reaction was performed at $95^{\circ} \mathrm{C}$ for $2 \mathrm{~min}$; and circulated 40 times at $95^{\circ} \mathrm{C}$ for $20 \mathrm{~s}, 50^{\circ} \mathrm{C}$ for $30 \mathrm{~s}$ and $60^{\circ} \mathrm{C}$ for $40 \mathrm{~s}$. The fluorescence were collected and recorded after finishing 40 cycles. And the reaction was performed on real time fluorescent quantitative PCR (Thermal Cycler). Data were analyzed using a relative critical threshold $(\mathrm{Ct})$ method where the amount of target normalized to the amount of inner control.

\subsection{Statistics}

Experimental data were expressed as mean \pm standard error. $T$ test was used for comparison samples between different two groups. Muti-sample comparison in the same group was analyzed by ANOVA. The SPSS 18.0

\begin{tabular}{cc} 
Table 1. Animal and grouping. \\
\hline Groups & NSS/qPCR \\
\hline TBI & 10 \\
Sham & 10 \\
TBI-Xuesaitong injection (via caudal vein) & 10 \\
Total & 30 \\
\hline
\end{tabular}


Table 2. Neurological Severity Scores (NSS).

\begin{tabular}{|c|c|}
\hline Motors tests & Points \\
\hline Raising rat by the tail & 3 \\
\hline \multicolumn{2}{|l|}{1 Flexion of forelimb } \\
\hline \multicolumn{2}{|l|}{1 Flexion of hindlimb } \\
\hline \multicolumn{2}{|l|}{1 Head moved $>10^{\circ}$ to vertical axis within $30 \mathrm{~s}$} \\
\hline Placing rat on the floor (normal $=0$; maximum $=3$ ) & 3 \\
\hline \multicolumn{2}{|l|}{0 Normal walk } \\
\hline \multicolumn{2}{|l|}{1 Inability to walk straight } \\
\hline \multicolumn{2}{|l|}{2 Circling toward the paretic side } \\
\hline \multicolumn{2}{|l|}{3 Fall down to the paretic side } \\
\hline Sensory tests & 2 \\
\hline \multicolumn{2}{|l|}{1 Placing test (visual and tactile test) } \\
\hline \multicolumn{2}{|c|}{2 Proprioceptive test (deep sensation, pushing the paw against the table edge to stimulate limb muscles) } \\
\hline Beam balance test $($ normal $=0$; maximum $=6$ ) & 6 \\
\hline \multicolumn{2}{|l|}{0 Balances with steady posture } \\
\hline \multicolumn{2}{|l|}{1 Grasps side of beam } \\
\hline \multicolumn{2}{|l|}{2 Hugs the beam and one limb falls down from the beam } \\
\hline \multicolumn{2}{|l|}{3 Hugs the beam and two limbs fall down from the beam, or spins on beam (>60s) } \\
\hline \multicolumn{2}{|l|}{4 Attempts to balance on the beam but falls off ( $>40 \mathrm{~s}$ ) } \\
\hline \multicolumn{2}{|l|}{5 Attempts to balance on the beam but falls off ( $>20 \mathrm{~s})$} \\
\hline \multicolumn{2}{|l|}{6 Falls off: No attempt to balance or hang on to the beam $(<20 s)$} \\
\hline Reflexes absent and abnormal movements & 4 \\
\hline \multicolumn{2}{|l|}{1 Pinna reflex (head shake when touching the auditory meatus) } \\
\hline \multicolumn{2}{|l|}{1 Corneal reflex (eye blink when lightly touching the cornea with cotton) } \\
\hline \multicolumn{2}{|l|}{1 Startle reflex (motor response to a brief noise from snapping a clipboard paper) } \\
\hline 1 Seizures, myoclonus, myodystony & \\
\hline Maximum points & 18 \\
\hline
\end{tabular}

Note: One point is awarded for the inability to perform the tasks or for the lack of a tested reflex; 13 to 18 indicates severe injury; 7 to 12 , moderate injury; 1 to 6 , mild injury.

software (IBM Company) was used for statistical analysis. A level of $\mathrm{P}<0.05$ was considered as statistical significant. When $\mathrm{P}<0.01$, it is considered as significant statistical difference.

\section{Results}

\subsection{NSS Scores}

The NSS Scores in TBI group and Xuesaitong treated group turned out to be a step-up trend compared with sham group. During 8 to $13 \mathrm{~d}$ after operation, there was significant difference in NSS scores within TBI group and Xuesaitong group respectively $(\mathrm{P}<0.01$ ). In these two groups, the NSS Scores had a tendency of rising over time after the operation up to $14 \mathrm{~d}$. It is implied that with the time passed following injury, rats got some extent of natural recovery. 
The NSS scores in TBI group was substantially higher than that of sham group $(\mathrm{P}<0.01)$. There was no marked difference for NSS scored between Xuesaitong group and TBI group $(P>0.05)$. NSS Scales in different experimental groups were shown in Table 3 and Figure 1.

\section{2. qPCR}

The level of Bax expression in TBI group was markedly higher than that of sham operative group and Xuesaitong treated group, respectively $(\mathrm{P}<0.01)$. The comparison of Bax expression level among TBI, Xuesaitong treated and sham operative group was shown in Table 4 and Figure 2.

Table 3. NSS Scales in different group.

\begin{tabular}{cccc}
\hline \multirow{2}{*}{ DPO } & \multicolumn{3}{c}{ NSS Scales (Mean \pm standard error) } \\
\cline { 2 - 4 } & TBI group & Xuesaitong group & Sham group \\
\hline 1 & $5.2707 \pm 0.61177$ & $4.3328 \pm 0.11655^{*}$ & $0.2462 \pm 0.03078^{*}$ \\
2 & $4.7017 \pm 0.20472$ & $4.6639 \pm 0.11755^{*}$ & $0.0586 \pm 0.00953^{*}$ \\
3 & $4.3627 \pm 0.11777$ & $4.2630 \pm 0.08558^{*}$ & $0.0000 \pm 0.00000^{*}$ \\
4 & $3.5687 \pm 0.05369$ & $3.5644 \pm 0.05255^{*}$ & $0.0000 \pm 0.00000^{*}$ \\
6 & $2.3539 \pm 0.08526$ & $2.1195 \pm 0.07089^{*}$ & $0.0000 \pm 0.00000^{*}$ \\
8 & $1.1968 \pm 0.03799$ & $0.8860 \pm 0.03101^{*}$ & $0.0000 \pm 0.00000^{*}$ \\
10 & $1.0003 \pm 0.03232$ & $0.8410 \pm 0.04815^{*}$ & $0.0000 \pm 0.00000^{*}$ \\
12 & $0.5975 \pm 0.01911$ & $0.5169 \pm 0.01374^{*}$ & $0.0000 \pm 0.00000^{*}$ \\
14 & $0.2272 \pm 0.02295$ & $0.0949 \pm 0.00402^{*}$ & $0.0000 \pm 0.00000^{*}$ \\
\hline
\end{tabular}

Note: ${ }^{*}$ Represented compared with TBI group. When $\mathrm{P}<0.01$, there was significant difference. DPO represents days post of the operation.

Table 4. BAX relative optical density scales.

\begin{tabular}{cc}
\hline Group & BAX \\
TBI & $0.0229 \pm 0.0024$ \\
TBI+XueSaiTong & $0.0175 \pm 0.001^{*}$ \\
Sham & $0.0115 \pm 0.002^{*}$ \\
\hline
\end{tabular}

Note: *Represented compared with TBI group, when $\mathrm{P}<0.01$, there was significant difference.

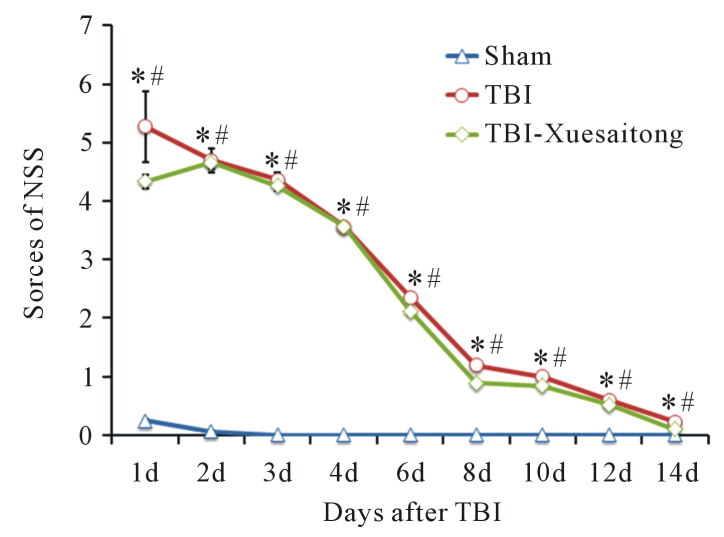

Figure 1. NSS Score polygram. * Represented comparison of inner groups in TBI and sham group. When $\mathrm{P}<0.05$ there was significant difference; "Represented intergroups comparison between TBI and Xuesaitong group. When $\mathrm{P}<0.05$, it was considered statistically significant. 


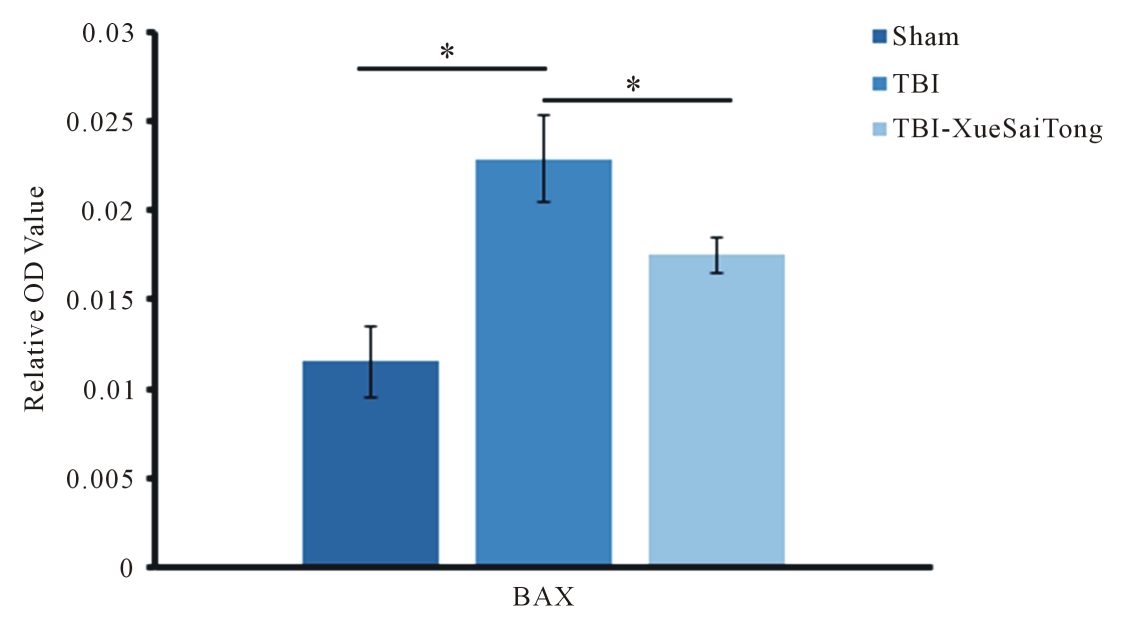

Figure 2. BAX relative optical density histogram. * Represented there was significant difference $(\mathrm{P}<0.01)$ between the two group connected with the transverse lines.

\section{Discussion}

Traumatic brain injury is one of the most common diseases in neurosurgical clinics with high incidence rate and mortality [1] [2]. In this study, NSS Score Evaluation revealed that there was marked difference between TBI and sham operative group, suggesting that the TBI rat models were successfully installed. While there was nearly not any significant difference between any two subgroups on the time spots from $8 \mathrm{~d}$ to $13 \mathrm{~d}$ after the operation in TBI group, it was implied that TBI rats had some certain ability to recover spontaneously. However, as there was obvious difference in NSS Scores between TBI and sham operative group, it was indicative that the injury resulted from TBI could not recover completely, that is, the rats of TBI had functional defect to a certain extent. The third, our results in NSS Score evaluation found that there existed a step-up trend in NSS Scores in TBI and Xuesaitong treated group when compared with sham group respectively, and the NSS scores in Xuesaitong treated group had no marked difference between those of TBI group, suggesting that Xuesaitong did not exhibited active role in the Neural functional recovery in TBI rats in this study.

Following brain injury, there was local ischemia occurred in the tissues surrounding the injury site. The local ischemia would further lead to local tissue necrosis, i.e. cellular apoptosis [3]. Real-time PCR showed that the level of Bax expression in TBI group was markedly considerably higher than that of sham operative group. It was strongly suggested that brain injury did led to the augmentation of Bax gene expression, which thereby resulted in the apoptosis of nerve cells. Previous studies found that Bcl-2/Bax ratio had a close association with cellular apoptosis. Notably, when Bax and Bcl-2 formed homo/heterodimer, it could play a crucial role in regulating cellular apoptosis. When Bax formed homodimer, it could result in cellular apoptosis, while when bcl-2 formed heterodimer, it could attenuate apoptotic rate of nerve cells by markedly suppress Bax, ultimately play a vital role in brain tissue protection [19]. It is concluded from above that when the content of heterodimer (Bcl-XL/Bax and Bcl2/Bax) above 50\% intracellularly, the cells commonly endure the apoptosis. Whereas when the content of Bax homodimer above $80 \%$ intracellularly, cellular apoptosis occurred [20]. Our results showed that the level of Bas expression in Xuesaitong treated group was markedly lower than that of TBI group. This demonstrated that Xuesaitong played roles in prohibiting Bax gene expression.

In summary, despite Xuesaitong could play its roles in brain tissue protection in brain injury as described in previous report [21], we found that it did not had any active and positive role in neural functional recovery when injected through caudal vein in TBI rats. Previous reported concluded that Xuesaitong may played its roles through suppressing Bax gene expression in that it not only could relieve the extent of brain edema and infarction area [22] [23], but also further ameliorate neural function, thereby ultimately contributing to the therapeutic effect of brain injury. However, currently, Xuesaitong was mostly used in the cerebral ischemia of rat models, even in clinical treatment, such as cerebral infarction therapy. Therefore, its concrete roles and associated underlying mechanism in brain injury await intensive studies. And the dose, pathway and timing for Xuesaitong administration are another important factors affecting its roles in brain protection. Furthermore, the development of brain injury may be involved in muti-mechanisms rather than a simple one. In this study, Bax gene expression 
was detected only on $1 \mathrm{~d}$ post of operation. Because of at this time, NSS Scores change among the three experimental groups was the biggest; we wanted to investigate the relationship of NSS Score-changes and Bas gene expression. Notably, the scope of timing for Bax detection should be enlarged in future studies so that more convincing outcomes can be obtained. Therefore, our study is a preliminary one. It is needed to intensively elucidate the concrete actions taken by Xuesaitong in therapy of brain injury. Importantly, more reliable evidence involved in changes of Bax gene expression and Xuesaitong's roles in brain injury awaits professional study.

\section{Acknowledgements}

This work was sponsored by the Associated Fund of science and technology Yunnan Government Agency with Kunming Medical University, 2015FB008, taken charge by Associate Professor Li Jin-Tao.

\section{References}

[1] Zhang, G. and Zhi, K.D. (2009) Mechanism of Cognitive Impairment Following Traumatic Brain Injury. Chin J Neuromed, 8, 637-639.

[2] Hao, S.-Y. and Liu, B.Y. (2005) Research Progress of Local Cerebral Ischemia Following Traumatic Brain Injury. Foreign Medical Sciences Section on Neurology \& Neurosurgery, 32, 430-434.

[3] Gan, Y., Xu, H.B. and Sun, X.B. (2007) Research Progress of Pharmacologic Actions of Panax Notoginsenosides. Li Shi Zhen Medicine and Materia Medical Research, 18, 1251-1252.

[4] Li, Z. and Cui, R.-Q. (2013) Research Progress on Roles of Injection of Chinese Drugs in the Therapy of Cardiovascular and Cerebrovascular Diseases. Traditional Chinese Medicine and Pharmacy of Nei Meng Gu, 32, 127-128.

[5] Zhao, X.-S. (2014) Experimental Study in Neuroprotective Roles of Panax Notoginsenosides in Rats with Cerebral Hemorrhage. Beijing Traditional Chinese Medicine and Pharmacy University, Beijing.

[6] Wang, Z.-J. (2013) Clinical Therapeutic Observation of 88 Cases Patients with Cerebral Thrombosi. Medical Research of China and Overseas, 11, 166.

[7] http://www.ebiotrade.com/emgzf/Merck200403/Untitled-2.htm

[8] Pecina-Slaus, N. (2009) Genetic and Molecular Insights into Apoptosis. Acta Medica Croatica, 2, 13-19.

[9] Kastelan, M., Massari, L.P. and Brajac, I. (2010) The Role of bcl-2 Family Proteins in Psoriasis. Lijec Vjesn, 132, 31-33.

[10] Tang, X.Y. and Pu, X.Y. (2011) Cloning and Expression of Mouse Bax Gene. Lab Med Clin, 8, 662-664.

[11] Ionoc, Y., Yamamoto, H., Krajewski, S., Reed, J.C. and Perucho, M. (2000) Mutational Inactivation of the Proapoptotic Gene BAX Confers Selective Advantage during Tumor Clonal Evolution. Proceedings of the National Academy of Sciences of the United States of America, 97, 10872-10877. http://dx.doi.org/10.1073/pnas.190210897

[12] Krajewska, M., Fenoglio-Preiser, C.M., Krajewski, S., Song, K., Macdonald, J.S., Stemmerman, G. and Reed, J.C. (1996) Immunohistochemical Analysis of Bcl-2 Family Proteins in Adenocarcrinomas of the Stomach. The American Journal of Pathology, 149, 1449-1457.

[13] Liu, J., Yin, S., Reddy, N., Spencer, C. and Sheng, S.J. (2004) Bax Mediates the Apoptosis-Sensitizing Effect of Maspin. Cancer Research, 64, 1703-1711. http://dx.doi.org/10.1158/0008-5472.CAN-03-2568

[14] Finnie, J.W. and Blumbergs, P.C. (2002) Traumatic Brain Injury. Veterinary Pathology, 39, 679-689. http://dx.doi.org/10.1354/vp.39-6-679

[15] Huo, Y.-Q. and Tang, Y.-F. (2007) A Kind of Brain Traumatic Model of Modified Following Weight. Journal of Guangxi Medical University, 24, 217-219.

[16] Chen, J.L., Li, Y., Wang, L. and Zhang, Z.G. (2001) Therapeutic Benefit of Intravenous Administration of Bone Marrow Stromal Cells after Cerebral Ischemia in Rats. Stroke, 32, 1005-1011. http://dx.doi.org/10.1161/01.STR.32.4.1005

[17] Germano, A.F., Dixon, C.E., d’Avella, D., Hayes, R.L. and Tomasello, F. (1994) Behavioral Deficits Following Experimental Subarachnoid Hemorrhage in the Rat. Journal of Neurotrauma, 11, 345-353. http://dx.doi.org/10.1089/neu.1994.11.345

[18] He, P., Zhu, D.-L., Zhu, P., Wang, R.-J. and Wang, X. (2012) The Effect of Total Panax Notoginseng Naponinsin on the Apoptosis of Brain Cells and the Expression of Apoptotic Protein Bax in Newbron Rat after Asphyxia. Journal of Clinical Pediatrics, 30, 1178-1181.

[19] Wang, W.D. and Chen, Z.T. (2007) Bel-2/Bax Ratio and the "Life or Death Fate" of Cells. Chinese Journal of Cancer Biotherapy, 14, 393-396. 
[20] Chen, Y., Constantini, S., Trembovler, V., Weinstock, M. and Shohami, E. (1996) An Experimental Model of Closed Head Injury in Mice: Pathophysiology, Histopathology, and Cognitive Deficits. Journal of Neurotrauma, 13, 557-568.

[21] Zhang, X.M., Wu, J.R. and Zhang, B. (2015) Xuesaitong Injection as One Adjuvant Treatment of Acute Cerebral Infarction: A Systematic Review and Meta-Analysis. BMC Complementary and Alternative Medicine, 15, 36. http://dx.doi.org/10.1186/s12906-015-0560-4

[22] Xi, W.-G. and Zhao, Z.-Y. (2009) Roles of PNS in Rat Brain Ischemia of Thrombogenicity. Chinese Herbs, 40, 234236.

[23] Qiao, C.-L., Ding, Y.-F. and Yang, C.-R. (2012) Pharmacologic Research Progress of Notoginseng Total Saponins. Modern Chinese Medicine, 14, 25-30. 